\title{
Empresas de referência na utilização do desdobramento da função qualidade
}

\author{
José Antonio Carnevalli - Dr. (UNIMEP) \\ jcarnevalli@hotmail.com \\ Paulo Augusto Cauchick Miguel - Ph.D. (USP) \\ cauchick@usp.br
}

\begin{abstract}
Desdobramento da Função Qualidade (QFD) é um importante método para desenvolver produtos que atendam e superem as expectativas dos clientes, por esta razão é importante realizar estudos sobre o uso deste método identificando os pontos chaves na sua implementação. Este artigo apresenta uma continuação da análise dos dados coletados por uma pesquisa tipo survey sobre o uso do QFD, realizada no Brasil, com a realização de entrevistas em algumas das empresas participantes da pesquisa, visando um maior aprofundamento do estudo. Para análise destes dados foram considerados oito critérios exemplares definidos pelos autores com base na literatura sobre o QFD. A análise das entrevistas revelou que existiam certas resistências de alguns funcionários ao uso do QFD, as quais devem ser superadas durante a aplicação. Também foram identificados aspectos exemplares no uso do método. Com os resultados, foi possível identificar os pontos chaves que devem ser considerados durante a implantação do QFD.
\end{abstract}

Palavras-chave: QFD; desenvolvimento de produto; planejamento da qualidade.

Quality Function Deployment (QFD) is an important method to develop products which attend and exceed customer's expectations; for this reason, it is important to study the use of this method in order to identify the key points in QFD implementation. This paper carries on the data analysis collected in a survey research on QFD carried out in Brazil, with interviews in some companies which participated in the survey, to make a deeper study of it. To analyze the data, the authors considered eight exemplary criterion with QFD literature support. Data interviews analysis showed that there were certain obstacles, posed by some employees, to the use of QFD which must be overcome during its application. Exemplary aspects on the use of the method were also identified. With the results, it was possible to identify the key points in the QFD implementation that should be considered.

Keywords: QFD; product development; quality planning.

\section{Introdução}

No mundo competitivo de hoje, torna-se essencial às empresas conseguirem a satisfação e a lealdade dos seus clientes para se manterem no mercado. Mas para atingir estes objetivos é necessário que a empresa conheça bem os seus clientes, identificando os seus requisitos mais importantes, que devem ser atendidos de modo satisfatório pelos produtos oferecidos por ela. Um dos métodos de desenvolvimento de produtos e serviços que consegue atingir estes objetivos é o Desdobramento da Função Qualidade (QFD). O QFD é um método de desenvolvimento de produtos que tem como propósito principal definir a garantia da qualidade na fase de projeto, além de identificar e traduzir as exigências dos clientes em características técnicas do produto para atender a essas exigências (AKAO, 1996; OHFUJI et al., 1997). Atualmente, o QFD é utilizado em vários países do mundo, tais como Reino Unido, Suécia, EUA e no Brasil, principalmente a partir do início da década de 90.

Mesmo após mais de dez anos de divulgação do método do QFD no Brasil, verifica-se uma escassez de dados sobre como é a sua aplicação no país. Procurando reduzir essa escassez de dados, foi realizado 
anteriormente um estudo tipo survey com os seguintes objetivos (ver MIGUEL, 2003; CARNEVALLI, et al., 2004): verificar o grau de introdução do QFD no Brasil, estudando principalmente as maiores empresas privadas, por faturamento, identificando quais são os benefícios, dificuldades de implantação e também empresas que já tenham maturidade no seu uso. Neste levantamento, verificou-se que as empresas têm dificuldades em implementar o método (ver MIGUEL, 2003; CARNEVALLI, et al., 2004). O presente trabalho é uma continuação da análise dos dados coletados na pesquisa tipo survey, incorporando dados e informações advindas de outras entrevistas realizadas posteriormente em algumas das empresas participantes do estudo. São discutidos aspectos exemplares da aplicação do QFD, que devem ser considerados pelas empresas que pretendem implantá-lo. Nos próximos tópicos são apresentados alguns exemplos da aplicação do QFD, um resumo da pesquisa tipo survey realizada no Brasil e dos métodos e técnicas de pesquisa utilizadas nas entrevistas, bem como seus respectivos resultados.

\section{Experiência na utilização do QFD}

A Tabela 1 apresenta alguns exemplos de aplicação do método do QFD, extraídos da literatura. Como pode-se verificar na tabela, o QFD é um método flexível, o que permite que seja utilizado no desenvolvimento ou re-projeto de uma vasta variedade de produtos. A sua principal característica é a capacidade de identificar requisitos prioritários para o projeto, sejam do cliente, requisitos ergonômicos ou ecológicos, dentre outros, para posteriormente traduzi-los em especificações do projeto visando atendêlos. Tal característica também tem incentivado a sua aplicação e a busca de melhoria contínua. Outra característica que se destaca na Tabela 1 é a capacidade do QFD de documentar o know how do projeto em suas matrizes, deste modo preservando este conhecimento para futuros projetos.

Tabela 1 - Exemplos da literatura do uso do QFD no desenvolvimento de Produtos.

\begin{tabular}{|c|c|}
\hline Autor & Aplicação para desenvolver produtos ecológicos \\
\hline $\begin{array}{l}\text { HALOG et } \\
\text { al., (2001) }\end{array}$ & $\begin{array}{l}\text { Halog et al. (2001) utilizam o QFD para otimizar produtos ecológicos considerando o } \\
\text { impacto ecológico do produto e as restrições de orçamento do projeto. Junto com o } \\
\text { QFD é utilizado um modelo matemático linear. Segundo Halog et al. (2001), com } \\
\text { pequenas modificações o QFD pode ser usado para melhoria continua e ser útil para } \\
\text { melhorar produtos considerando aspectos ambientais. }\end{array}$ \\
\hline $\begin{array}{l}\text { MASUI et } \\
\text { al., (2003) }\end{array}$ & $\begin{array}{l}\text { Masui et al. (2003) desenvolvem uma metodologia (modelo conceitual) para aplicar o } \\
\text { QFD a projeto de produtos considerando requisitos de consciência ambiental no início } \\
\text { de projeto do produto. O QFD foi eficiente para considerar aspectos ambientais no } \\
\text { desenvolvimento do produto e apresentar várias soluções para melhorar o produto, as } \\
\text { quais foram avaliadas. }\end{array}$ \\
\hline $\begin{array}{l}\text { RAHIMI \& } \\
\text { WEIDNER, } \\
\text { (2002) }\end{array}$ & $\begin{array}{l}\text { Rahimi \& Weidner (2002) uniram conceitos ambientais nas matrizes do QFD. Nessa } \\
\text { matrizes as qualidades exigidas envolvem requisitos dos clientes, requisitos ecológicos } \\
\text { e de custos. }\end{array}$ \\
\hline Autor & Aplicação para re-projetar produto \\
\hline $\begin{array}{l}\text { CHAO \& } \\
\text { ISHII, } \\
\text { (2004) }\end{array}$ & $\begin{array}{c}\text { Chao \& Ishii (2004) desenvolveram uma metodologia baseada no QFD para } \\
\text { minimizar falhas no projeto, podendo também ser usada para revisão de projetos } \\
\text { atuais. Foram feitas aplicações acadêmicas e um estudo de caso. Segundo os autores o } \\
\text { QFD ajuda a identificar erros de projeto, a avaliar subsistemas, ajuda na definição dos } \\
\text { objetivos do projeto, a documentar os dados do projeto e a administrar os riscos de } \\
\text { falha (CHAO \& ISHII, 2004). }\end{array}$ \\
\hline LIN \& WEI & Lin \& Wei (2005) desenvolveram um modelo de programação linear para ajudar na \\
\hline
\end{tabular}




\begin{tabular}{|c|c|}
\hline$(2005)$ & $\begin{array}{l}\text { otimização de projetos de melhorias de produto, buscando reduzir custos e tempo. } \\
\text { Apresentam uma aplicação na melhoria de mouse do computador, sendo que a } \\
\text { aplicação foi acadêmica e não foi introduzida na indústria. Somente utilizaram } \\
\text { princípios do QFD para definir as qualidades exigidas e as características da } \\
\text { qualidade. }\end{array}$ \\
\hline $\begin{array}{l}\text { MARSOT, } \\
\text { (2005) }\end{array}$ & $\begin{array}{l}\text { Marsot (2005) apresenta um estudo de caso onde o QFD foi utilizado para re-projetar } \\
\text { uma faca de desossar considerando aspectos ergonômicos. Esta é uma das pesquisas } \\
\text { francesas que busca integrar aspectos ergonômicos nos produtos chamado de CEROM } \\
\text { (Conception ERgonomique d'Outils à Main). Os benefícios do uso do QFD são } \\
\text { identificar as melhores soluções entre as qualidades exigidas dos clientes e qualidades } \\
\text { exigidas ergonômicas, e conseguir o consenso no atendimento dos dois tipos, além de } \\
\text { alterar as características da qualidade atuais do produto para atender as qualidades } \\
\text { ergonômicas exigidas. }\end{array}$ \\
\hline Autor & Aplicações diversas no desenvolvimento de produtos \\
\hline $\begin{array}{l}\text { COSTA et } \\
\text { al., (2001) }\end{array}$ & $\begin{array}{c}\text { Revisão bibliográfica sobre o uso do QFD na indústria de alimentos. Os autores } \\
\text { destacam os seguintes benefícios do QFD: tomada de decisão em consenso com a } \\
\text { equipe; desdobramento simultâneo devido ao uso da equipe ser multifuncional } \\
\text { (COSTA et al., 2001). }\end{array}$ \\
\hline $\begin{array}{l}\text { MIGUEL et } \\
\text { al., (2003) }\end{array}$ & $\begin{array}{l}\text { Miguel et al. (2003) apresentam a aplicação e implementação do QFD numa empresa } \\
\text { fabricante de filmes flexíveis. Segundo os autores, o uso do QFD trouxe os seguintes } \\
\text { benefícios: sistematizou o processo de desenvolvimento; melhoria da comunicação; } \\
\text { democratizou o conhecimento entre os membros da equipe, a capacidade de } \\
\text { planejamento e registrou o conhecimento da empresa. }\end{array}$ \\
\hline $\begin{array}{c}\text { HSIAO \& } \\
\text { LIU, (2005) }\end{array}$ & $\begin{array}{l}\text { Os autores desenvolveram um sistema de suporte inteligente para projetar famílias de } \\
\text { produtos. Esse sistema utiliza o QFD para identificar as qualidades exigidas e as } \\
\text { características da qualidade e usa o ISM (Interpretive Structural Model) para verificar } \\
\text { a hierarquia dos componentes e como eles se relacionam com o produto final (análise } \\
\text { das características da qualidade versus características da qualidade). O benefício do } \\
\text { uso do QFD foi identificar os principais requisitos dos clientes para cada tipo de } \\
\text { mercado e as principais mudanças no re-projeto do produto para atender os clientes. }\end{array}$ \\
\hline $\begin{array}{c}\text { SANFORD, } \\
\text { (2005) }\end{array}$ & $\begin{array}{l}\text { O autor participou da equipe de estudantes que utilizaram o QFD para ajudar a } \\
\text { desenvolver um robô, para uma competição robótica. O QFD ajudou a identificar as } \\
\text { características de qualidade críticas (SANFORD, 2005). }\end{array}$ \\
\hline
\end{tabular}

A seguir é apresentado um resumo das etapas de aplicação do QFD, adaptado de Cheng et al. (1995), Akao (1996) ê Ohfuji et al. (1997):

1) A empresa define no planejamento estratégico o plano anual de desenvolvimento de produto e com este plano define-se o produto a ser desenvolvido;

2) Selecionar os membros da equipe do QFD. Segundo Ohfuji et al. (1997), os membros da equipe variam com as etapas, mas o líder da equipe deve ser fixo;

3) Realizar treinamento sobre o método: treinamentos devem considerar aspectos práticos e de implementação, mas a empresa deve adequar a implantação à sua realidade específica (OHFUJI et al., 1997);

4) Realizar pesquisa de mercado com o público alvo, para identificar seus requisitos para o produto a ser desenvolvido. Ohfuji et al. (1997) também recomendam o uso de informações internas, como dados sobre as reclamações dos clientes;

5) Definir o modelo conceitual, que são as tabelas e matrizes do QFD que serão feitas para atingir os objetivos do projeto. 
6) Elaborar a matriz da qualidade: nesta etapa são identificados os requisitos dos clientes prioritários (qualidade planejada), tradução destes requisitos (qualidade exigida) em especificações de projeto (características da qualidade) e definição dos valores destas especificações (qualidade projetada), de modo a satisfazer os clientes e atingir os objetivos do projeto;

7) Realizar as demais tabelas e matrizes definidas no modelo conceitual. Segundo Akao (1996), os valores das especificações de projeto (qualidade projetada) devem ser desdobrados até que seja possível garantir a qualidade do projeto; os tipos de desdobramentos são:

a) Desdobramento da Qualidade pode envolver o desdobramento das especificações de projeto em funções, subsistemas, componentes, processos, etc.;

b) Desdobramento da Tecnologia: verifica se a empresa tem condições de fabricar produtos com os valores definidos no Desdobramento da Qualidade. Caso contrário, verificar quais são as possíveis soluções e a viabilidade do projeto;

c) Desdobramento dos Custos: definir o custo objetivo do produto (AKAO, 1996). Utilizando o grau de importância dos itens definidos no Desdobramento da Qualidade é possível identificar os custos objetivos das funções, subsistemas, componentes (CHENG et al., 1995). Com esta análise é possível verificar os casos em que os custos são maiores que o previsto e propor soluções para a sua redução;

d) Desdobramento da Confiabilidade: identificar os componentes e processos críticos nos quais se deve aplicar FMEA para prevenir e reduzir a ocorrência de falhas.

8) Realizar o QFDr (Desdobramento da Função Qualidade no sentido restrito) envolve o desdobramento das funções de trabalho para garantir a qualidade, para definir quais são as atividades do desenvolvimento do produto a serem realizadas. Para desenvolver o QFDr, Cheng et al. (1995) recomendam o uso do diagrama em árvore e do método 5W1H;

9) Com os resultados da aplicação do QFD são elaboradas Tabelas de Fluxograma do Processo, Tabela de Garantia da Qualidade e de Padrões Técnicos de Processos, de modo que seja possível fabricar o produto com as especificações definidas.

As empresas devem avaliar a necessidade de seguir estas etapas quando decide implementar o QFD; para Lowe \& Ridgway (2000a) o método deve ser adaptado à empresa. A seguir é apresentado um resumo dos resultados da pesquisa tipo survey realizada anteriormente.

\subsection{Resultados da pesquisa tipo survey}

Os objetivos da pesquisa tipo survey foram os seguintes: avaliar o grau de introdução do QFD no Brasil, estudando principalmente as maiores empresas privadas, de forma a identificar os benefícios e dificuldades de implementar este método, bem como as empresas que já têm experiência no seu uso (CARNEVALLI et al., 2004). Nesta pesquisa, foi enviado um questionário pelo correio para 506 empresas, que faziam parte da amostra. A taxa de retorno foi de 21\% (106 empresas), onde se verificou que pouco mais de $18 \%$ utilizava o método (20 empresas). Entretanto, verificou-se que uma empresa fez apenas um estudo teórico do método, não o introduzindo, reduzindo o número para 19 empresas utilizando QFD (17,9\% do total), mostrando que o QFD não era muito utilizado. Segundo os resultados da pesquisa (CARNEVALLI et al., 2004; MIGUEL, 2003), estas empresas iniciaram o uso do QFD buscando principalmente melhorias no processo de desenvolvimento de produtos, e iniciaram a implantação após a metade da década de 90, mostrando que o uso do método no Brasil ainda era relativamente recente. Sobre as dificuldades de implantação do QFD, as que mais se destacaram neste estudo foram: a falta de experiência no método; falta de comprometimento dos membros da equipe de trabalho do QFD e dificuldades de se trabalhar com matrizes muito grandes. Em relação aos benefícios, destacaram-se: o aumento da satisfação dos clientes, melhoria do trabalho em grupo e da comunicação entre os departamentos. Também foram identificadas por Carnevalli et al. (2004), cinco empresas com experiência em QFD (mais de 26\% das que usam), que poderiam ser consideradas como referência no uso do método no país. Após a conclusão da survey, foi feito um aprofundamento dos dados com a realização de 
entrevistas em algumas das empresas que participaram da survey, sendo apresentados os resultados de quatro delas.

\section{Métodos e técnicas das entrevistas e da definição de aspectos exemplares do uso do QFD}

Durante as entrevistas foi utilizado um formulário que se poderia classificar como sendo semi-estruturado, com questões abertas (dissertativas). Isto porque, apesar de ser um formulário padronizado, onde o entrevistador tem uma série de questões para serem feitas seguindo um roteiro, este tem características de um formulário estruturado segundo Lakatos \& Marconi (1991). O entrevistador também tinha a liberdade de formular novas questões, alterar as ordens das perguntas e permitir ao entrevistado falar à vontade, características de um formulário não-estruturado, como destacado por Lakatos \& Marconi (1991). As entrevistas foram agendadas com antecedência, sendo entrevistadas as mesmas pessoas que participaram da pesquisa tipo survey, que responderam o questionário daquele estudo e, em alguns casos, também junto com a equipe de projeto que aplicou o QFD. As entrevistas tiveram duração média de duas horas e o seu registro foi feito com o uso de gravador, sendo também feitas anotações por um dos entrevistadores. Após a transcrição das entrevistas, a equipe de pesquisa enviou o arquivo por e-mail para os entrevistados verificarem se havia alguma informação sigilosa, para a empresa, que deveria ser retirada, além de validar a precisão das informações prestadas. A equipe de pesquisa também se comprometeu a não divulgar o nome da empresas entrevistadas sem a sua devida autorização. Posteriormente o conteúdo das entrevistas foi re-analisado visando identificar informações sobre expectativas iniciais na introdução do QFD dentro da empresa, sendo estas expectativas importantes para se entender objetivos de aplicação e possíveis resistências ao seu uso por parte de seus funcionários.

Na seqüência, é apresentada a definição dos critérios que foram considerados para identificar os aspectos exemplares do uso do QFD em empresas que participaram da pesquisa tipo survey no Brasil.

\subsection{Critérios exemplares do uso do QFD}

Foram considerados como critérios exemplares: a experiência das empresas no uso do método; (data de início da implantação); o apoio da alta administração; duração do treinamento em QFD; número de pessoas na equipe; freqüência e duração das reuniões; forma de obtenção dos requisitos do cliente; uso de outros desdobramentos, além da matriz da qualidade, avaliação da implantação e seus custos; e manutenção de registros de melhorias alcançadas. Estes critérios são descritos a seguir.

- Ano de início da implantação: foi considerado como critério de experiência em QFD para os casos das empresas que já tinham seis anos de aplicação do método. A definição de seis anos como critério de experiência em QFD foi baseada na experiência japonesa sobre o uso do QFD. Segundo Akao et al. (1987), no Japão leva-se dois anos para o QFD ser sistematizado pelas empresas e seis anos para que realmente o método seja incorporado como prática cotidiana.

- Ter o apoio da alta administração: este critério foi considerado importante por ser citado em vários artigos da literatura como pré-requisito para aplicar o QFD (CHIN et al., 2001; KENGPLO, 2004; LAGER, 2005; POLITIS, 2005) e a falta deste apoio também é citado como uma das causas que geram dificuldades no uso do método (CRISTIANO et al., 2000; GOVERS, 2001; MARTINS \& ASPINWALL, 2001; MIGUEL, 2003; GINN \& ZAIRI, 2005). Isto porque o QFD causa alterações no modo de se trabalhar dentro das empresas, as quais só serão aceitas se contar com o apoio da alta administração.

- Treinamento em QFD: este critério foi considerado importante pois verificou-se, em Miguel (2003) e em Carnevalli et al. (2004), que uma das principais dificuldades de se implantar o QFD é 
a falta de experiência no método. Além disso, a literatura também cita como dificuldades do uso do QFD a falta de treinamento no método (LOWE \& RIDGWAY (2000a); KENGPLO, 2004; GINN \& ZAIRI, 2005). Por esta razão, a duração e realização de um treinamento em QFD é um importante aspecto para tentar minimizar esta dificuldade. Foi considerado como aspecto exemplar empresas que realizam treinamento com duração próxima de cinco dias, como recomendado por Cheng et al. (1995).

- Número de pessoas que participaram das equipes do QFD: Lowe \& Ridgway (2000a) recomendam o uso de equipes pequenas para facilitar o consenso da equipe durante as reuniões, por esta razão foram considerados como critérios exemplares as empresas que se enquadram nos critérios de Ohfuji et al. (1997), ou seja, de 5 ou 6 pessoas. Isto porque, segundo Ohfuji et al. (1997), o ideal seria equipes com essa quantidade aproximada de participantes, pois equipes com menos de 4 pessoas podem tornar-se pouco produtivas por ficarem sobrecarregadas, e equipes de mais de 7 pessoas podem ser improdutivas por causarem muitas discussões atrasando os trabalhos.

- Freqüência e duração das reuniões: são importantes pois como a maioria dos trabalhos de aplicação do QFD é realizado durante estas reuniões. A freqüência e a sua duração estão diretamente ligados ao tempo de desenvolvimento do projeto com o uso do método. Lowe \& Ridgway (2000a) recomendam que o método e as reuniões do QFD devem ser adaptadas à realidade da empresa e aos objetivos do projeto. Como critérios relevantes considerou-se como relevante as empresas que realizam reuniões diárias ou semanais. Sobre a duração destas reuniões do QFD foram consideradas como exemplares as que duravam de meio dia a um dia inteiro, demonstrando grande comprometimento com o uso do método.

- Forma de se obter os requisitos do cliente: foi considerado como um critério muito importante, pois, segundo Lowe \& Ridgway (2000b), identificar os verdadeiros requisitos do segmento de mercado alvo é fator chave para o sucesso do QFD. Isto porque, caso a empresa não consiga identificar corretamente estes requisitos, os resultados da aplicação do QFD poderão ser negativos, pois desenvolverá um produto que não satisfará os clientes. Neste critério, com base em Cristiano et al. (2000), considerou-se como prática exemplar as empresas que estão utilizando informações externas (consulta por telefone, entrevistas, grupo de foco, questionários via correio) bem como internas (informações fornecidas por vendas, relatório de reclamações, relatos de clientes internos, uso de dados de garantia), pois deste modo as empresas ouvem diretamente as exigências dos seus clientes, ao mesmo tempo que utiliza dados internos, como, por exemplo, informações de atendimento ao cliente, para aperfeiçoar seus produtos.

- Realização de outros desdobramentos, além da matriz da qualidade: este critério foi considerado como aspecto exemplar porque a matriz da qualidade apenas define a qualidade projetada que 0 produto deve ter para satisfazer o cliente, mas não garante que a empresa possa fabricar tal produto com essa qualidade. Por esta razão, é necessário que as empresas verifiquem quais são as outras matrizes que devem ser feitas para que consigam fabricar o produto com os valores da qualidade projetada. Na pesquisa de Carnevalli et al. (2004), verificou-se que as empresas que realizam outras matrizes, além da matriz da qualidade, conseguem mais benefícios do QFD e tem um maior índice de sucesso na implantação do método. Cheng (2003) recomenda fazer um modelo conceitual para definir as matrizes e tabelas necessárias para desenvolver produtos que atendam os requisitos dos clientes.

- Avaliação da implantação, avaliação dos custos de implantação e manutenção de registros de melhorias alcançadas: são critérios que foram considerados como aspectos exemplares por serem 
práticas muito importantes para confirmar a eficácia no uso do método. Entretanto, esta avaliação não é fácil de ser realizada, pois o sucesso do projeto com o uso do QFD costuma ser avaliado de forma subjetiva pelos participantes (CHENG, 2003).

\section{Aspectos exemplares do uso do QFD identificados nas empresas participantes da survey}

Na pesquisa tipo suvery sobre o uso do QFD no Brasil, foram identificadas 14 empresas, do total de 19, que apresentaram pelo menos um aspecto exemplar de aplicação do método. Esse tópico cita alguns aspectos exemplares das 10 empresas que não foram entrevistadas, sendo que as 4 entrevistadas são apresentadas no tópico seguinte. Como na pesquisa foram encontradas mais de uma empresa operando no mesmo setor, elas foram numeradas como empresa (1), (2) e assim por diante.

A Empresa de Eletrodomésticos (1) apresenta os seguintes aspectos exemplares: experiência (mais de dez anos de implantação do método); tamanho da equipe do QFD (cinco pessoas); freqüência das reuniões (semanais); duração das reuniões (em torno de um dia). Além disso, esta empresa realiza outras matrizes além da matriz da qualidade.

Na Empresa de Eletrodomésticos (2) destacam-se os aspectos exemplares: freqüências das reuniões (diárias); realização de outros desdobramentos (não se restringe à matriz da qualidade), forma de se obter os requisitos do cliente (usa informações internas e externas).

Na Empresa de Eletrodomésticos (3) foram encontrados os seguintes critérios exemplares: tamanho da equipe (cinco participantes); freqüências das reuniões do uso do método (semanais); realização de outros desdobramentos, além da matriz da qualidade e obtenção dos requisitos dos clientes com informações internas e externas.

A Empresa de Produtos Químicos (1) tem os seguintes aspectos exemplares: forma de se obter os requisitos do cliente (usa informações internas e externas); realiza outros desdobramentos, além da matriz da qualidade; avalia a implantação, seus custos e mantém registros das melhorias alcançadas com o QFD.

Na Empresa de Produtos Químicos (2) se destacam os critérios: tempo de experiência em QFD (por ter mais de sete anos de implantação); realizar outras matrizes, além da matriz da qualidade e obter os requisitos dos clientes com informações internas e externas à empresa.

Na Empresa Automobilística os critérios que se destacam são: experiência em QFD (ter iniciado a implantação há seis anos); tamanho da equipe do QFD (seis pessoas em média); avaliação dos custos da implantação do QFD e manutenção de registro das melhorias alcançadas com o método.

A Empresa de Máquinas e Equipamentos atende um critério: manutenção de registro de melhorias alcançadas com o método.

Na Empresa de Produtos e Serviços Ferroviários se destacam os critérios: freqüência das reuniões (semanais); forma de se obter os requisitos do cliente (usa informações internas e externas); realização de outros desdobramentos (não se restringe à matriz da qualidade); e manutenção de registro de melhorias alcançadas com o uso do QFD.

A Empresa de Metalúrgica Básica atende os seguintes critérios: treinamento (mais de três dias de duração); duração das reuniões do QFD (em torno de meio dia); realização de outras matrizes, além da matriz da qualidade. 
Na Empresa de Material de Construção se destacam três critérios: freqüência das reuniões (semanais); obtenção dos requisitos do cliente com informações internas e externas; e manutenção de registro de melhorias alcançadas com o método.

\section{Aspectos exemplares do uso do QFD identificados nas empresas entrevistadas}

Neste tópico são apresentadas algumas das práticas exemplares da implantação e do uso do QFD, identificadas nas 4 empresas entrevistadas.

\subsection{Empresas do setor de alimentos}

Os critérios exemplares identificados nesta empresa são: apoio da alta administração; experiência em QFD (mais de nove anos de aplicação); realização de outros desdobramentos além da matriz da qualidade; obtenção dos requisitos dos clientes (uso de informações internas e externas); duração das reuniões do QFD (em torno de meio dia). Esta empresa tem mais de dez projetos concluídos.

Na implantação do QFD, a empresa de alimentos contou com um grande apoio da alta administração. O objetivo inicial da empresa na introdução do método era se tornar líder em desenvolvimento de produtos. Chin et al. (2001) e Lager (2005) consideram que o apoio da alta administração é um pré-requisito para aplicar o método. Guimarães (2003) e Parkin et al. (2002) também consideram muito importante definir claramente os objetivos do uso do QFD para o sucesso da sua aplicação.

Antes de implantar o QFD a empresa acionou uma consultoria e verificou a necessidade de implantar primeiro a garantia da qualidade, para dar suporte ao método. Isto porque é importante que a empresa tenha um controle da variabilidade do seu processo de fabricação, mantendo as características do produto o mais próximo possível das especificações definidas pelo QFD. Isso está em conformidade com a literatura pois, de acordo com Govers (1996; 2001), para se ter sucesso na implantação do QFD é necessário que a empresa já tenha um nível de qualidade, em relação ao controle de problemas de modo sistemático. Esse aspecto também é confirmado no trabalho de Okes (2002) que aponta o QFD como um método a ser utilizado por empresas com um alto nível de maturidade em qualidade.

Após implantada a garantia da qualidade, iniciou-se o treinamento para aplicar o QFD. O treinamento do método foi realizado por uma consultoria externa, que realizou exercícios práticos onde o QFD era aplicado em produtos da própria empresa. Ginn \& Zairi (2005) também recomendam treinamento prático do QFD como importante para sua implantação. Este tipo de treinamento é estritamente necessário pois é somente com a aplicação, mesmo simulada, que aparecem e são resolvidas a maioria das dúvidas sobre o método.

Para a implantação do QFD, a empresa decidiu realizar um projeto piloto de um dos produtos já fabricados por ela, contando para isto com o apoio da mesma consultoria que realizou o treinamento no método. Segundo Cheng et al. (1995), é importante iniciar a implantação do método com um produto que a empresa já tenha o know how, pois senão a equipe do projeto piloto enfrentará duas dificuldades, pois entender o método do QFD e desenvolver o know how necessário para fabricar o produto dificulta o sucesso da implantação. Para Govers (1996), é importante que a equipe tenha o apoio de um facilitador que conheça o método e tenha habilidade em construir e dirigir a equipe.

Para formar a equipe multifuncional do QFD, a empresa escolheu as melhores pessoas de cada setor envolvido para o desenvolvimento do projeto piloto. Outros aspectos exemplares para o desenvolvimento do projeto piloto desenvolvido por esta empresa foram: realização do modelo conceitual, desdobrando o produto até o chão de fábrica e avaliação do protótipo usando a tabela da qualidade exigida, convidando os clientes para prová-lo. 
Segundo a empresa, o uso do QFD trouxe a necessidade de investimentos em infra-estrutura, com a construção de um laboratório sensorial, sendo muito importante para a indústria do setor de alimentos, conseguir quantificar a qualidade exigida pelo cliente. Este fato também é confirmado na aplicação do QFD em outra indústria alimentícia, onde também foi necessário montar um laboratório sensorial (PAIVA \& CHENG, 2001).

Após a conclusão do projeto piloto, a empresa buscou diminuir o tamanho das matrizes do QFD nos projetos subseqüentes. Para isto, utilizou-se o QFD em partes separadas do produto ao invés do produto completo, como, por exemplo, o recheio de alguns pratos. Chou (2004) também recomenda dividir o projeto em grupos de sub-projetos, de modo a analisar os dados separadamente em várias matrizes pequenas. Esta foi uma solução encontrada pela empresa para sanar uma das dificuldades mais citadas na literatura sobre a aplicação do método: o tamanho grande das matrizes (BOUCHEREAU \& ROWLANDS, 2000a; b; SHIN \& KIM, 2000; HAN et al., 2001; MIGUEL, 2003; CARNEVALLI et al., 2004; CHOU, 2004; DIKMEN et al., 2005; MARSOT, 2005).

Para o entrevistado, os pontos chaves para implantação do QFD são:

1. Decisão da alta administração com apoio financeiro;

2. Formar uma equipe excelente;

3. Ter um apoio de uma assessoria externa para o treinamento e ajudar no projeto piloto.

Com a re-análise da transcrição da entrevista verificaram-se as seguintes expectativas iniciais da aplicação do método:

A empresa buscava tornar-se líder no desenvolvimento de produto usando o QFD para garantir a qualidade. Entretanto a empresa tinha inicialmente um conhecimento superficial do QFD, limitado à matriz da qualidade. Com a realização de treinamento, por uma consultoria externa, a empresa ganhou uma visão completa do método verificando a necessidade de desenvolver um modelo conceitual para garantir que a voz do consumidor chegasse até o chão de fábrica, de modo a atingir o objetivo estabelecido para o uso do QFD. A literatura (CHENG, 2003) também recomenda a elaboração do modelo conceitual pelos mesmos motivos.

Durante a realização do projeto piloto, a alta administração estava mais preocupada em conseguir introduzir o QFD na empresa do que os benefícios gerados pelo piloto, o que pode indicar um forte comprometimento da sua parte na introdução do método. Entretanto, para garantir a continuidade do uso do método na empresa, o líder da equipe também estava preocupado em atingir resultados satisfatórios com esta aplicação, para incentivar o seu uso futuro. O projeto piloto atendeu os dois objetivos: introduziu o QFD na empresa e atingiu bons resultados com a melhoria do produto.

Durante a introdução também foram identificadas algumas resistências de alguns departamentos ao uso do método. Estas foram superadas com a ajuda do apoio da alta administração, comprovando a sua importância como pré-requisito no uso do método.

- $\quad$ Também foi identificada uma dificuldade a ser superada, segundo a empresa. Tanto a diretoria como os funcionários têm um primeiro impacto (negativo) ao visualizar pela primeira vez uma matriz do QFD. Segundo o entrevistado, as pessoas se assustam com a matriz e os símbolos das correlações, por não saberem os seus significados, prejulgando o método como complicado, antes de receberem uma explicação mais detalhada. 


\subsection{Empresas fornecedoras de filmes flexíveis para embalagens}

Esta empresa apresenta os seguintes critérios exemplares: apoio da alta administração; freqüências das reuniões (semanais); duração das reuniões (duas horas, podendo chegar a um dia inteiro, se necessário); obtenção dos requisitos dos clientes (uso de informações internas e externas); realização de outras matrizes, além da matriz da qualidade, e de estar iniciando o desdobramento dos custos. No total, esta empresa vem aplicando o QFD em 11 projetos.

A empresa decidiu usar o método do QFD para dar suporte aos seus projetos de desenvolvimento de produto. Como a empresa é certificada pela norma ISO 9001, desde 1994, ela já tinha, como no caso da empresa do setor de alimentos, o suporte necessário para implementar o método que, por sua vez, também auxiliou a empresa a cumprir os novos requisitos da revisão de 2000 da série ISO 9000. Segundo Govers (2001), a série ISO 9000 ajuda a empresa a atingir as especificações de projeto definidas pelo QFD. Como ocorreu na empresa citada anteriormente, a implantação do QFD contou com o apoio da alta administração, sendo realizada num projeto piloto para aperfeiçoar um produto já fabricado pela empresa. Chen \& Weng (2003) afirmam que a equipe terá mais dificuldades na elaboração do QFD nos casos de desenvolver um produto totalmente novo com o método. Segundo Cheng et al. (1995), a empresa deve escolher para o projeto piloto um dos produtos que ela já fabrique, que seja importante para a empresa e que necessite de melhorias.

Também como ocorreu no caso da empresa do setor de alimentos, a equipe do projeto piloto era formada pelos melhores funcionários das áreas envolvidas com o projeto. Algumas recomendações da literatura para a seleção da equipe são: montar equipes experientes (DIKMEN et al., 2005); com poucos membros para facilitar o consenso durante as reuniões; formada por pessoas positivas focadas nos clientes (LOWE \& RIDGWAY, 2000a) e escolher membros que estejam comprometidos com o método (LAGER, 2005). Um dado importante sobre a equipe é que, segundo o respondente, não existe um número ideal de participantes, que varia conforme a etapa de aplicação do método. O importante é que em cada etapa estejam presentes as pessoas certas, que tenham o conhecimento necessário para realizar aquela etapa.

Para dar apoio às equipes do QFD a alta administração contou com suporte externo para realizar o treinamento e ajudar na implantação do método. Segundo a empresa, a presença de um facilitador externo durante a implantação do QFD foi muito importante, pois possibilitou a consolidação do conhecimento do método a partir do momento que as dúvidas iam surgindo na aplicação e sendo discutidas pela equipe. Deste modo, a empresa diminuiu uma das dificuldades muito citadas na literatura, a falta de conhecimento em QFD (MARTINS \& ASPINWALL, 2001; MIGUEL, 2003; MIGUEL et al., 2003; CARNEVALLI et al., 2004; GINN \& ZAIRI, 2005).

Para coletar os requisitos dos clientes, a empresa usou entrevistas e informações fornecidas por vendas. Segundo Lower \& Ridgway (2000b), identificar os verdadeiros requisitos do segmento de mercado alvo é fator chave para o sucesso do QFD. Um aspecto exemplar na implantação do piloto foi a elaboração de um manual sobre o QFD, contendo todas as informações necessárias para apoiar futuras aplicações do método na empresa.

A equipe do projeto piloto realizava reuniões semanais, com o apoio externo, para elaboração do projeto. Após a conclusão do projeto piloto, a empresa iniciou outros projetos com o uso do QFD, tendo nestas novas equipes alguns membros do primeiro projeto para multiplicar o conhecimento adquirido. Esta é uma recomendação citada por Cheng et al. (1995). Até o momento, foram 5 projetos já desenvolvidos e 6 estão em desenvolvimento usando o QFD.

Segundo as entrevistas, os pontos chaves na implantação do QFD são: 
1. Apoio da alta administração;

2. Desenvolver o Know How em QFD;

3. Convencer as pessoas da importância do uso do QFD, para eliminar as resistências e conseguir a colaboração de todos. Segundo Ginnn \& Zairi (2005), o treinamento em QFD deve também buscar este objetivo: conscientizar sobre a importância do uso do método.

Com a re-análise da transcrição desta entrevista foram identificadas a seguintes expectativas no uso do QFD:

- $\quad$ Democratizar o conhecimento de desenvolvimento de produto pela empresa: isto porque em muitos casos este conhecimento estava restrito a um só departamento ou a apenas a algumas pessoas da empresa. Paiva \& Cheng (2001) também citam a democratização e documentação do conhecimento na equipe como um dos benefícios identificados em pesquisa ação realizada em uma pequena empresa de alimentos.

- $\quad$ Entender as correlações das características de qualidade com os processos produtivos e os seus efeitos no produto final;

Atingir resultados em curto prazo: a expectativa inicial da empresa era que o QFD reduzisse o tempo de projeto em $75 \%$; um dos membros da equipe esperava uma redução de $50 \%$. O projeto piloto reduziu o tempo de projeto em $50 \%$. Apesar de estar fora da expectativa da empresa, o QFD já gerou uma redução do tempo de projeto considerado como adequado. A redução no tempo de projeto é um benefício muito citado na literatura (BOUCHERAU \& ROWLANDS, 2000a; LEE \& KO, 2000; LOWE \& RIDGWAY, 2000a; KARSAK et al., 2002; RAMASAMY \& SELLADURAI, 2004).

- $\quad$ A equipe que utiliza o QFD também tinha como expectativa que o método reduzisse o número de alterações no projeto, o que foi atingido. Este benefício também costuma ser muito citado pela literatura (por exemplo, LIU, 2000; LOWE \& RIDGWAY, 2000a; RAMASAMY \& SELLADURAI, 2004), além de ser um dos motivos pelo qual o QFD foi criado, o que explica por que a empresa tinha esta expectativa.

\subsection{Empresas de máquinas e equipamentos}

São critérios exemplares desta empresa: experiência em QFD (mais de 12 anos de uso do método); treinamento (quatro dias e meio de duração); apoio da alta administração; obtenção dos requisitos dos clientes (uso do método do QFD para desenvolver um produto plataforma mundial, sendo que a equipe do QFD envolveu profissionais do mundo todo e ouviu clientes de vários países) e manutenção de registro das melhorias alcançadas com o método.

Sendo uma grande empresa multinacional, decidiu usar o QFD para desenvolver um produto que atendesse vários dos seus mercados (EUA, Europa, América Latina). Por esta razão, um fator interessante nesta aplicação do QFD foi a existência de várias equipes trabalhando separadamente, para coletar as exigências dos seus clientes em cada mercado. Com os resultados desta aplicação do QFD, foi desenvolvido um produto plataforma e seus respectivos derivativos para atender as diferentes exigências de cada mercado.

As equipes foram treinadas no centro de treinamento na matriz da empresa. A duração deste treinamento foi de quatro dias e meio, o que está dentro do recomendado por Cheng et al. (1995), ou seja, treinamento em torno de cinco dias. Um aspecto exemplar de destaque nesta aplicação é o fato da equipe que vai utilizar o QFD passar também por um treinamento de quatro horas sobre o trabalho em equipes multifuncionais. Neste treinamento, os membros da equipe são convencidos das vantagens do trabalho em equipes multifuncionais em relação ao trabalho de forma seqüencial. Segundo a empresa, este treinamento 
é necessário para dar apoio à aplicação do QFD. A literatura (KATHAWAL \& MOTWANI, 1994; DAMANTE, 1997) também recomenda a realização deste treinamento sobre o trabalho em grupo e gestão de trabalhos. Guimarães (2003) também recomenda que a equipe do QFD seja não só treinada no método, mas que tenha consciência sobre os objetivos e expectativas do projeto, o cronograma e as suas responsabilidades.

A alta administração da empresa deu muito apoio no desenvolvimento do projeto, sendo que alguns anos depois da implantação, a empresa facilitou a comunicação direta com a administração, tendo sido criado um cargo de gerente responsável para cada projeto que a fábrica desenvolve. Este gerente responde diretamente para os diretores da fábrica, sem ter que se submeter a outra área da empresa. Segundo Damante (1997), ter apoio da administração, e acompanhamento e cobrança do projeto pelos altos níveis administrativos são fatores de sucesso para aplicar o QFD. Para Politis (2003), este apoio é critico para aplicar o QFD.

Também foram encontradas as seguintes expectativas no uso do QFD, após a re-análise desta entrevista: contribuição do QFD para diminuir os riscos do projeto, a partir do momento que a empresa identifica os requisitos dos clientes e as características da qualidade prioritárias para atendê-los.

\subsection{Empresas automobilísticas}

Esta empresa apresenta os seguintes critérios exemplares: treinamento (cinco dias de duração); número de pessoas na equipe do QFD (em torno de seis pessoas); freqüências das reuniões (semanais); duração das reuniões (de uma a duas horas, podendo chegar a um dia inteiro se necessário). Também é um aspecto exemplar desta empresa conseguir aplicar o QFD em um produto complexo como são os veículos automobilísticos.

A empresa iniciou o uso do QFD buscando um método para verificar se as exigências do mercado, identificadas pela empresa internamente, estavam corretas, e usar o método para traduzi-las em características do produto. Um fato interessante é que este motivo para iniciar o uso do QFD coincide com uma das definições do método muito citada na literatura (DIJKSTRA \& BIJ, 2002; FRANCESCHINI \& ROSSETTO, 2002; MIGUEL et al., 2003; RAHIM \& BAKSH, 2003; GONZALEZ et al., 2004a; LORENZO et al., 2004; RAMASAMY \& SELLADURAI, 2004; OMACHONU \& BARACH, 2005; YAN et al., 2005), apesar de limitar o QFD a uma das suas funções, "a tradução das necessidades dos clientes em características do projeto", o que pode estar influenciando e limitando a sua aplicação a apenas este aspecto.

A implantação e o treinamento do QFD foi realizado por uma consultoria externa. Segundo a empresa, o melhor treinamento é aquele que trabalha a aplicação do QFD dentro de um produto da empresa, pois treinamento somente teórico não supre as dúvidas e dificuldades que só aparecerão durante a aplicação do método. A empresa realizou os dois tipos de treinamento: primeiro um somente teórico de cinco dias e depois um novo treinamento realizado tendo como base uma aplicação do QFD, que ajudou a solidificar os conceitos. Como já apresentado anteriormente, este tipo de treinamento é importante para reduzir dificuldades causadas por falta de conhecimento no uso do QFD.

Na implantação do QFD, a equipe teve todo o apoio necessário da administração mais direta da fábrica, pois sendo uma empresa multinacional, a alta administração está mais interessada nos resultados conseguidos com o produto desenvolvido, não se envolvendo na escolha do método utilizado para atingir estes resultados.

Um aspecto exemplar da aplicação do QFD nesta empresa é que a freqüência e duração das reuniões das equipes do QFD são definidas em função da necessidade e da demanda do trabalho de cada projeto, 
podendo, em alguns casos e em determinadas etapas, durar até o dia todo, mas sendo, em média, reuniões semanais com duração de uma a duas horas. Govers (1996) defende a necessidade das empresas darem às equipes do QFD tempo suficiente para estabelecer e dirigir suas atividades, o que está de acordo com a recomendação de Lowe \& Ridgway (2000a) já apresentada: as reuniões do QFD devem ser adaptadas à realidade da empresa e aos objetivos do projeto. Para garantir o comprometimento da equipe que aplica o método, esta equipe também participa do planejamento das atividades e da definição dos prazos do projeto.

Após um ano do lançamento do produto é realizada uma avaliação deste produto para verificar se os objetivos iniciais do projeto foram atingidos. Outro aspecto exemplar da aplicação do QFD é que sempre que a empresa inicia um novo projeto com o uso do método, a equipe responsável por este projeto tem um re-treinamento de um a dois dias para revisarem todos os conceitos do QFD.

Esta empresa está identificando os requisitos dos clientes através de relatórios de reclamações e informações fornecidas por vendas e usa as entrevistas para definir o grau de importância que cada requisito tem para o seu cliente.

Com a re-análise dos dados da entrevista de 2005 verificou-se que a equipe ficou um pouco assustada no início do projeto com a complexidade do QFD, mas o considerava como uma importante ferramenta para atender as necessidades dos clientes no projeto. A complexidade do método é uma das dificuldades do uso do QFD citada na literatura (HAN et al., 2001; CHIEN \& SU, 2003; GINN \& ZAIRI, 2005).

Também foram identificadas algumas resistências internas ao uso do QFD, as quais foram superadas com os resultados positivos do projeto piloto. Um membro da equipe que utilizou o QFD em um projeto fora do piloto relatou algumas resistências encontradas no uso do QFD. Segundo ele alguns membros da equipe não acreditavam no método e por isto não concordaram em realizar parte da matriz da qualidade, a análise comparativa dos valores de especificações técnicas dos produtos dos concorrentes com o da empresa (características da qualidade). Essas pessoas argumentavam que esta etapa da matriz era muito cara, apesar de ser uma etapa importante para a empresa definir valores de especificações de projeto que atendam os clientes e superem os concorrentes. Segundo o entrevistado, nem todos entendiam os objetivos do QFD, por esta razão a resistência ao seu uso.

Tabela 2 - Aspectos relevantes do uso do QFD identificados em 14 empresas participantes da survey

\begin{tabular}{|c|c|c|c|c|c|c|c|c|c|c|}
\hline Critérios exemplares & 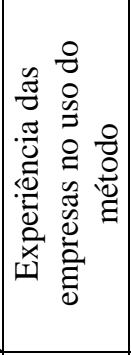 & 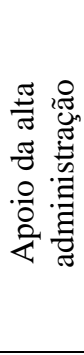 & 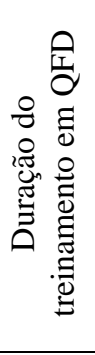 & 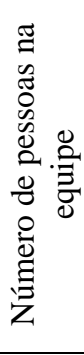 & 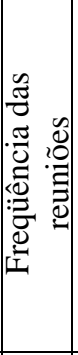 & 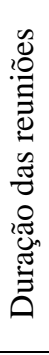 & 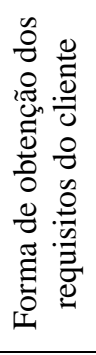 & 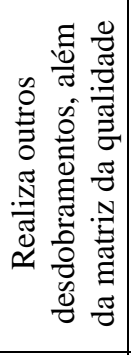 & 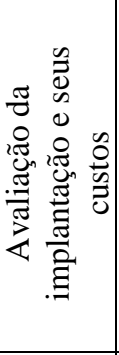 & 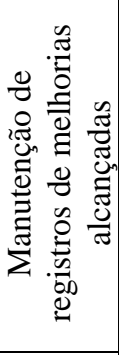 \\
\hline Empresa do setor de alimentos* & $\mathrm{X}$ & $\mathrm{X}$ & & & & $\mathrm{X}$ & $\mathrm{X}$ & $\mathrm{X}$ & & \\
\hline Empresa de Eletrodomésticos (1) & $\mathrm{X}$ & & & $\mathrm{X}$ & $\mathrm{X}$ & $\mathrm{X}$ & & $\mathrm{X}$ & & \\
\hline Empresa de Eletrodomésticos (2) & & & & & $\mathrm{X}$ & & $X$ & $\mathrm{X}$ & & \\
\hline Empresa de Eletrodomésticos (3) & & & & $\mathrm{X}$ & $\mathrm{X}$ & & $\mathrm{X}$ & $\mathrm{X}$ & & \\
\hline $\begin{array}{l}\text { Empresa fornecedora de filmes } \\
\text { flexíveis para embalagens* }\end{array}$ & & $\mathrm{X}$ & & & $\mathrm{X}$ & $\mathrm{X}$ & $X$ & $\mathrm{X}$ & & \\
\hline Empresa de Produtos Químicos (1) & & & & & & & $\mathrm{X}$ & $\mathrm{X}$ & $\mathrm{X}$ & $\mathrm{X}$ \\
\hline Empresa de Produtos Químicos (2) & $\mathrm{X}$ & & & & & & $\mathrm{X}$ & $\mathrm{X}$ & & \\
\hline Empresa Automobilística (1) & $\mathrm{X}$ & & & $X$ & & & & & $X$ & $X$ \\
\hline Empresa Automobilística (2)* & & & $\mathrm{X}$ & $\mathrm{X}$ & $\mathrm{X}$ & $\mathrm{X}$ & & & & \\
\hline Empresa de Máquinas e & & & & & & & & & & $X$ \\
\hline
\end{tabular}




\begin{tabular}{|c|c|c|c|c|c|c|c|c|c|c|}
\hline Equipamentos (1) & & & & & & & & & & \\
\hline $\begin{array}{c}\text { Empresa de máquinas e } \\
\text { Equipamentos (2)* }\end{array}$ & $\mathrm{X}$ & $\mathrm{X}$ & $\mathrm{X}$ & & & & $\mathrm{X}$ & & & $\mathrm{X}$ \\
\hline $\begin{array}{c}\text { Empresa de Produtos e Serviços } \\
\text { Ferroviários }\end{array}$ & & & & & $\mathrm{X}$ & & $\mathrm{X}$ & $\mathrm{X}$ & & $\mathrm{X}$ \\
\hline Empresa de Metalúrgica Básica & & & $\mathrm{X}$ & & & $\mathrm{X}$ & & $\mathrm{X}$ & & \\
\hline \\
Empresa de Material de Construção & & & & & $\mathrm{X}$ & & $\mathrm{X}$ & & & $\mathrm{X}$ \\
\hline
\end{tabular}

\section{Conclusões}

Analisando os critérios e aspectos exemplares apresentados pelas 14 empresas, verificou-se que os pontos chaves que devem ser considerados pelas empresas que desejam implantar o QFD com sucesso são:

1. A empresa deve desenvolver um nível de controle de qualidade que permita fabricar produtos com as especificações determinadas pelo QFD. Caso não tenha este nível de qualidade, ela deve verificar a possibilidade de desenvolvê-lo. Caso contrário, o uso do QFD não alcançará todo o seu potencial, limitando os seus benefícios, pois a empresa não conseguirá produzir produtos com todas as especificações desejadas.

2. Ter o apoio da alta administração. Este apoio é necessário devido às mudanças nas relações de trabalho que o QFD pode trazer.

3. A empresa deve desenvolver o conhecimento necessário para implementar o método. Quando a empresa não tem este conhecimento, ela deve buscar o apoio necessário que ajude no treinamento e na implantação. Além disso, deve-se registrar todo o conhecimento gerado nos projetos num manual, que poderá servir para orientar futuras aplicações do método. O treinamento também deve buscar conscientizar as pessoas envolvidas com o QFD (equipe e departamento envolvidos) sobre a importância do uso do QFD, para eliminar as resistências e conseguir a colaboração de todos.

4. A equipe multifuncional do projeto piloto deve ser formada pelas pessoas mais competentes de cada área envolvida no projeto, além de serem participantes que acreditem no método e serem formadoras de opinião. As equipes devem ter em torno de 6 membros, buscando evitar de se trabalhar com equipes muito pequenas ou muito grandes. Entretanto, o número de membros na equipe pode variar de etapa para etapa, mas as pessoas chaves com as informações necessárias para a realização de cada etapa devem estar presentes. Os membros da equipe do projeto piloto devem apoiar e orientar as novas equipes, transferindo sua experiência.

5. A empresa deve buscar introduzir o método através de um projeto piloto, num produto que ela já tenha know how, e que necessite de melhoria.

6. A freqüência e duração das reuniões do projeto com o QFD devem ser conforme a demanda dos trabalhos, podendo durar um dia inteiro em algumas etapas. Seria importante que estas reuniões ocorressem pelo menos semanalmente, de forma que se tenha uma continuidade no uso do método.

7. Deve-se utilizar informações externas (por exemplo realizar entrevistas com os clientes) e internas (por exemplo usar relatórios de reclamações) para identificar os seus requisitos, pois deste modo a empresa verifica o que os clientes realmente desejam ao consultá-los, ao mesmo tempo que utilizando, por exemplo, dados de garantia, pode aperfeiçoar os seus produtos.

8. A empresa não deve apenas realizar a matriz da qualidade, mas desenvolver um modelo conceitual onde ela identifique todos os desdobramentos necessários para fabricar produtos com os valores da qualidade projetada. No entanto, cada desenvolvimento deve considerar a quantidade e tipo de matrizes envolvidas. 
9. A empresa deve buscar meios de avaliar a implantação do QFD, seus custos e deve manter os registros das melhorias alcançadas com o QFD, para poder confirmar a eficácia no uso do método. Após o lançamento do produto desenvolvido com o QFD, fazer um acompanhamento para verificar se os objetivos iniciais do projeto foram atingidos no mercado.

Com a releitura das entrevistas foi possível verificar que as empresas iniciaram o uso do QFD buscando atingir os benefícios citados na literatura, tais como: garantir a qualidade do produto; reduzir o tempo de desenvolvimento e o número de alterações no projeto; traduzir os requisitos dos clientes em especificações do projeto do produto para atendê-los e reduzir o risco de projeto; democratizar o conhecimento e preservar o know how da empresa. Também foram identificadas algumas resistências internas ao uso do método os quais a empresa deve buscar meios de superar, em alguns casos o apoio da alta administração ou da gerência tem sido fundamental para superar estas resistências.

Continuando este trabalho, está sendo realizada uma pesquisa que pretende fazer um estudo metodológico sobre o QFD, que diminua suas dificuldades de implantação e permita às empresas usufruírem de todos os seus benefícios. Para isto pretende-se desenvolver um modelo sistêmico de aplicação do QFD, com base no projeto axiomático.

\section{Agradecimentos}

Os autores agradecem as seguintes instituições, sem as quais não seria possível a realização desse trabalho: à FAPESP, ao CNPq, ao FAP - Fundo de Apoio à Pesquisa da UNIMEP através do projeto SEAC 207/99 e à CAPES. Também agradecem às empresas que possibilitaram a realização das entrevistas.

\section{Referências}

AKAO, Y. Introdução ao desdobramento da qualidade. Belo Horizonte: Fundação Christiano Ottoni, 1996. $187 \mathrm{p}$.

AKAO, Y. et al. Surveys and reviews on Quality Function Deployment in Japan. In: PROCEEDINGS OF THE INTERNATIONAL CONFERENCE FOR QUALITY CONTROL, JUSE and IAQ, Tokyo, 1987 p. 171-6.

BOUCHEREAU; V.; ROWLANDS,H. Methods and techniques to help quality function deployment (QFD). Benchmarking: An International Journal, v.7, n.1, p. 8-16, 2000a.

BOUCHEREAU, V.; ROWLANDS, H. Quality function deployment: the unused tool. Engineering Management Journal, v.10, n.1, p. 45-52, 2000b.

CARNEVALLI, J. A. et al. Aplicação do QFD no desenvolvimento de produtos: levantamento sobre seu uso e perspectivas para pesquisas futuras. Gestão \& Produção, v.11, n.1, p. 33-49, 2004.

CHAO, L.P.; ISHII, K. Project quality function deployment. International Journal of Quality \& Reliability Management, v.21, n.9, p. 938-958, 2004.

CHEN, L.H.; WENG, M.C. A fuzzy model for exploiting quality function deployment. Mathematical And Computer Modelling. v.38, n.5-6, p. 559-570, 2003.

CHENG, L. C., et al. QFD: planejamento da qualidade. Belo Horizonte: Fundação Christiano Ottoni, 1995. 261p. 
CHENG, L.C. QFD in product development: methodological characteristics and a guide for intervention. International Journal of Quality \& Reliability Management, v.20, n.1, p. 107-122, 2003.

CHIEN, T.K.; SU, C.T. Using the QFD concept to resolve customer satisfaction strategy decisions. International Journal of Quality \& Reliability Management, v.20, n.3, p. 345-359, 2003.

CHIN, K.S. et al. A quality function deployment approach for improving technical library and information services: a case study. Library Management, v.22, n.4/5, p. 195-204, 2001.

CHOU, S.M. Evaluating the service quality of undergraduate nursing education in Taiwan - using quality function deployment. Nurse Education Today, v.24, n.4, p. 310-318, 2004.

COSTA, A.I.A. et al. Quality function deployment in the food industry: a review. Food Science \&Technology, v.11, n.9-10, p. 306-314, 2001.

CRISTIANO, J.J. et al. Customer-driven product development through quality function deployment in the U.S. and Japan. Journal of Product Innovation Management, v.17, n.4, p. 286-308, 2000.

DAMANTE, F.C. Desdobramento da Função Qualidade: um estudo de sua aplicação no Brasil. 162p. Dissertação (Mestrado em Administração) - Faculdade de Economia, Administração e Contabilidade, Universidade de São Paulo, São Paulo, 1997.

DIKMEN, I. et al. Strategic use of quality function deployment (QFD) in the construction industry. Building and Environment, v.40, n.2, p. 245-255, 2005.

DIJKSTRA, L.; BIJ, H.V.D. Quality function deployment in healthcare: Methods for meeting customer requirements in redesign and renewal. International Journal of Quality \& Reliability Management, v.19, n.1, p. 67-89, 2002.

EKDAHL, F.; GUSTAFSSON, A. QFD: the Swedish experience. In: THE NINTH SYMPOSIUM ON QUALITY FUNCTION DEPLOYMENT, 9., Novi, 1997. Anais... Novi: Michigan, 1997, p.15-27.

FRANCESCHINI, F.; ROSSETTO, S. QFD: an interactive algorithm for the prioritization of product's technical design characteristics. Integrated Manufacturing Systems, v.13, n.1, p. 69-75, 2002.

GINN, D.; ZAIRI, M. Best practice QFD application: an internal/external benchmarking approach based on Ford Motors' experience. International Journal of Quality \& Reliability Management, v.22, n.1, p. 3858, 2005.

GONZALEZ; M.E. et al. QFD strategy house: an innovative tool for linking marketing and manufacturing strategies. Marketing Intelligence \& Planning, v.22, n.3, p. 335-348, 2004.

GOVERS, C. P. M. What and how about quality function deployment (qfd). International journal of production economics, v.46-47, p. 575-585, December, 1996.

GOVERS, C.P.M. QFD not just a tool but a way of quality management. International Journal of Production Economics, v.69, n.2, p.151-159, 2001.

GUIMARÃES, I.M. QFD, analisando seus aspectos culturais organizacionais. Banas Qualidade, v.12, n.128, p. 56-66, 2003. 
HALOG, A. et al. Using quality function deployment for technique selection for optimum environmental performance improvement. Journal of Cleaner Production, v.9, n.5, p. 387-394, 2001.

HAN, S.B. et al. A conceptual QFD planning model. International Journal of Quality \& Realiability Management, v.18, n.8, p. 796-812, 2001.

HSIAO, S.W; LIU, E. A structural component-based approach for designing product family. Computers in Industry, v.56, n.1, p. 13-28, 2005.

KATHAWALA, Y.; MOTWANI, J. Implementing Quality Function Deployment. Research and Concepts, v.6, n.6, p. 31-35, 1994.

KARSAK, E.E. et al. Product planning in quality function deployment using a combined analytic network process and goal programming approach. Computers \& Industrial Engineering, v.44, n.1, p. 171-190, 2002.

KENGPOL, A. Quality Function Deployment (QFD) in Small to Medium-sized Enterprises: A Study of Obstacles in Implementing QFD in Thailand. International Journal of Management, v.21, n.3, p. 393-402, 2004.

LAGER, T. The industrial usability of quality function deployment: a literature review and synthesis on a meta-level. R\&D Management, v.35, n.4, p. 409-426, 2005.

LAKATOS, E.M; MARCONI, M.A. Fundamentos de metodologia científica. 3. ed. São Paulo: Atlas, 1991. $270 \mathrm{p}$.

LEE, S.F.; KO, A.S.O. Building balanced scorecard with SWOT analysis, and implementing "Sun Tzu's The Art of Business Management Strategies" on QFD methodology. Managerial Auditing Journal, v.15, n.1/2, p. 68-76, 2000.

LIN, S.J; WEI, C.C. A Study on the Linear Programming in Time Cost Analysis of Product Improve Design- a Focus on Computer Mouse Products. Journal of American Academy of Business, v.7, n.2, p. 182-186, 2005.

LIU, X.F. Software quality function deployment. Potentials, IEEE, v.19, n.5, p. 14-16, 2000.

LORENZO, S. et al. Análisis matricial de la voz del cliente: QFD aplicado a la gestión sanitaria. Gaceta Sanitaria, v.18, n.6, p. 464-471, 2004.

LOWE, A.; RIDGWAY, K. UK user's guide to quality function deployment. Engineering Management Journal, v.10, n.3, p. 147-155, 2000a.

LOWE, A.J.; RIDGWAY, K. Optimization Impossible? : The importance of customer segmentation in quality function deployment. Quality Progress, v.33, n.7, p. 59-64, 2000b.

MARSOT, J. QFD: a methodological tool for integration of ergonomics at the design stage. Applied Ergonomics, v.36, n.2, p. 185-192, 2005.

MARTINS, A. \& ASPINWALL, E. M. Quality function deployment: an empirical study in the UK. Total Quality Management, v.12, n.5, p. 575-588, 2001. 
MASUI, K. et al. Applying Quality Function Deployment to environmentally conscious design.

International Journal of Quality \& Reliability Management, v.20, n.1, p. 90-106, 2003.

MIGUEL, P.A.C. The state-of-the-art of the Brazilian QFD applications at the top 500 companies. International Journal of Quality \& Reliability Management, v.20, n.1, p. 74-89, 2003. 